\title{
Article \\ Promoter Methylation of PRKCB, ADAMTS12, and NAALAD2 Is Specific to Prostate Cancer and Predicts Biochemical Disease Recurrence
}

\author{
Kristina Daniunaite ${ }^{1}$, Arnas Bakavicius ${ }^{2,3,4}$, Kristina Zukauskaite ${ }^{2}$, Ieva Rauluseviciute ${ }^{1}$, \\ Juozas Rimantas Lazutka ${ }^{1}$, Albertas Ulys ${ }^{2}$, Feliksas Jankevicius ${ }^{2,3,4}$ and Sonata Jarmalaite ${ }^{1,2, *}$ \\ 1 Life Sciences Center, Institute of Biosciences, Vilnius University, 10257 Vilnius, Lithuania; \\ vailaomalyn@yahoo.com (K.D.); ieva.rauluseviciute@gmail.com (I.R.); juozas.lazutka@gf.vu.lt (J.R.L.) \\ 2 National Cancer Institute, 08660 Vilnius, Lithuania; arnas.bakavicius@gmail.com (A.B.); \\ kristinazu@gmail.com (K.Z.); albertasulys@gmail.com (A.U.); Feliksas.Jankevicius@santa.lt (F.J.) \\ 3 Centre of Urology, Vilnius University Hospital Santaros Klinikos, 08661 Vilnius, Lithuania \\ 4 Faculty of Medicine, Vilnius University, 03101 Vilnius, Lithuania \\ * Correspondence: sonata.jarmalaite@nvi.lt; Tel.: +370-5-2190901; Fax: +370-5-2720164
}

check for

updates

Citation: Daniunaite, K.; Bakavicius, A.; Zukauskaite, K.; Rauluseviciute, I.; Lazutka, J.R.; Ulys, A.; Jankevicius, F.; Jarmalaite, S. Promoter Methylation of PRKCB, ADAMTS12, and $N A A L A D 2$ Is Specific to Prostate Cancer and Predicts Biochemical Disease Recurrence. Int. J. Mol. Sci. 2021, 22, 6091. https://doi.org/ $10.3390 /$ ijms 22116091

Academic Editor: Michele Caraglia

Received: 6 April 2021

Accepted: 1 June 2021

Published: 5 June 2021

Publisher's Note: MDPI stays neutra with regard to jurisdictional claims in published maps and institutional affiliations.

Copyright: (c) 2021 by the authors. Licensee MDPI, Basel, Switzerland. This article is an open access article distributed under the terms and conditions of the Creative Commons Attribution (CC BY) license (https:/ / creativecommons.org/licenses/by/ $4.0 /)$.

\begin{abstract}
The molecular diversity of prostate cancer (PCa) has been demonstrated by recent genomewide studies, proposing a significant number of different molecular markers. However, only a few of them have been transferred into clinical practice so far. The present study aimed to identify and validate novel DNA methylation biomarkers for PCa diagnosis and prognosis. Microarraybased methylome data of well-characterized cancerous and noncancerous prostate tissue (NPT) pairs was used for the initial screening. Ten protein-coding genes were selected for validation in a set of $151 \mathrm{PCa}, 51 \mathrm{NPT}$, as well as 17 benign prostatic hyperplasia samples. The Prostate Cancer Dataset (PRAD) of The Cancer Genome Atlas (TCGA) was utilized for independent validation of our findings. Methylation frequencies of ADAMTS12, CCDC181, FILIP1L, NAALAD2, PRKCB, and ZMIZ1 were up to $91 \%$ in our study. PCa specific methylation of ADAMTS12, CCDC181, NAALAD2, and $P R K C B$ was demonstrated by qualitative and quantitative means (all $p<0.05$ ). In agreement with PRAD, promoter methylation of these four genes was associated with the transcript down-regulation in the Lithuanian cohort (all $p<0.05$ ). Methylation of ADAMTS12, NAALAD2, and PRKCB was independently predictive for biochemical disease recurrence, while NAALAD2 and PRKCB increased the prognostic power of multivariate models (all $p<0.01$ ). The present study identified methylation of ADAMTS12, NAALAD2, and PRKCB as novel diagnostic and prognostic PCa biomarkers that might guide treatment decisions in clinical practice.
\end{abstract}

Keywords: prostate cancer; biochemical recurrence; DNA methylation; ADAMTS12; NAALAD2; $P R K C B$

\section{Introduction}

Despite tremendous improvements in diagnostics and treatment tactics, prostate cancer (PCa) is the second most common malignancy among men and the leading cause of cancer-related death among all urogenital cancers [1]. Timely diagnosis of the disease is essential for selecting the most appropriate treatment strategy and successful disease management. After introducing aggressive prostate-specific antigen (PSA) testing into clinical practice, the diagnosis of PCa has dramatically shifted towards the early stage and localized disease [2]. However, limited PSA prognostic power has increased the detection of clinically insignificant disease, leading to over-treatment with a huge negative impact on males' physical and mental well-being [3]. All these drawbacks encouraged the search for new diagnostic and prognostic PCa biomarkers with better performance characteristics.

Aberrant promoter methylation is an early event in carcinogenesis and has been extensively studied in the development of PCa [4]. Methylation of promoter region has 
been reported to be responsible for silencing more than 100 PCa genes, where glutathione Stransferase pi 1 (GSTP1) and RAS association domain family member 1 (RASSF1) has been the most intensively analyzed [4]. Recent genome-wide studies have revealed novel insight into the epigenetic landscape of $\mathrm{PCa}$ [5-9] and suggested new diagnostic and prognostic biomarkers. However, only a small part of them have been transferred into clinical practice so far $[5,7,8]$. Scientific evidence about prognostic DNA methylation biomarkers remains scarce as most investigations have resulted in ambiguous data. Besides, most of these studies have focused on comparing cancerous with noncancerous prostate tissue (NPT) rather than focusing on disease aggressiveness. However, aberrant DNA methylation is a promising source for PCa biomarkers, so thorough screening and consecutive validation in independent cohorts are needed to identify the most prospective ones for clinical usage.

The present study aimed at the identification and validation of novel DNA methylation biomarkers for PCa diagnosis and prognosis. Initially, 10 protein-coding genes were selected from previously reported microarray-based methylome screening [10] for promoter methylation analysis in PCa and control samples. For validation, a 2-step approach was used utilizing the Prostate Cancer Dataset (PRAD) of The Cancer Genome Atlas (TCGA) as an independent validation cohort [9]. Methylated promoter status was further associated with downregulated gene expression and clinico-pathological characteristics of PCa patients. Survival analysis was performed to evaluate the prognostic potential of the epigenetic biomarkers.

\section{Materials and Methods}

\subsection{Patients and Samples}

Treatment-naïve patients $(N=151)$ with histologically confirmed PCa who underwent radical prostatectomy (RP) at Vilnius University Hospital Santaros Klinikos between January 2008 and December 2014 were included. Cancerous $(N=151)$ and noncancerous prostate tissue $(N=51)$ samples were collected from the definitive pathology of these patients. As a control group, 17 benign prostatic hyperplasia (BPH) samples, obtained from open prostatectomy material due to $\mathrm{BPH}$, were included in the study. All tissues were dissected and evaluated by an expert pathologist, as reported previously [11]. Approval from the Lithuanian Bioethics Committee was obtained, and written informed consent was obtained from all participants.

Biochemical disease recurrence $(\mathrm{BCR})$ following $\mathrm{RP}$ was defined as a postoperative PSA $>0.2 \mathrm{ng} / \mathrm{mL}$ with a subsequent confirmatory value [12]. Full follow-up data were available for $88.7 \%(134 / 151)$ of patients with a mean follow-up of 3.3 years. Clinicopathological and molecular characteristics of the study subsets are provided in Table S1.

\subsection{DNA Methylation Microarray Data}

For the screening step, genome-wide DNA methylation profiling data of 9 paired $\mathrm{PCa}$ and NPT samples (GEO identifier GSE89243), reported in our previous study [10], has been reanalyzed to identify potential PCa biomarkers. Samples were processed according to the manufacturer's protocol using two-color Human DNA Methylation $1 \times 244 \mathrm{~K}$ Microarrays, which interrogate 27,627 known CpG islands (Agilent Technologies, Santa Clara, CA, USA). Saturated, non-uniform, and outlier probe signals were excluded from the analysis. The Cy3/Cy5 fluorescence ratios representing methylated/reference DNA were calculated and normalized to obtain $\log _{2}$ values for further analysis. Probe annotations were uploaded from the SureDesign platform (https: / / earray.chem.agilent.com/suredesign; accessed 1 March 2018). They were updated using the UCSC Genome Browser (https:/ / genome.ucsc. edu; accessed 1 March 2018) according to the latest version of the human reference genome (GRCh38). Probes that were not detected in $\geq 30 \%$ of all samples were filtered out. After additional group comparison-specific filtering, only probes detected in $100 \%$ of samples in at least 1 of 2 groups were compared. Fold change (FC) values were estimated, and a paired (where applicable) or unpaired t-test was applied. Calculations were performed with GeneSpring GX v14.5 software (Agilent Technologies). 
The gene set enrichment analysis (GSEA) was performed using the publicly accessible online GSEA tool and Molecular Signatures Database (MSigDB, v5.2; http:/ / software. broadinstitute.org/gsea; accessed 1 March 2018) [13]. The collection of 50 hallmark gene sets, which conveys a specific biological state or process and displays the coherent expression, were utilized for GSEA [14]. False discovery rate (FDR) q-value with the cut-off $<0.05$ was used to correct for multiple testing.

\subsection{DNA Purification and Qualitative Methylation Analysis}

DNA was extracted from fresh-frozen tissue samples following the standard phenolchloroform purification protocol as described previously [10]. Four hundred ng of purified DNA were bisulfite-modified, using the EZ DNA Methylation ${ }^{\mathrm{TM}}$ Kit (Zymo Research, Irvine, CA, USA) following the manufacturer's instructions, and analyzed by methylationspecific PCR (MSP). MSP primers, overlapping with the location of the microarray probes of interest, were designed with Methyl Primer Express Software v1.0 (Applied Biosystems ${ }^{\mathrm{TM}}$, Thermo Fisher Scientific, Carlsbad, CA, USA) and ordered from Metabion (Martinsried, Germany) (Table S2). The reaction mix $(25 \mu \mathrm{L})$ consisted of $1 \times$ Maxima Hot Start Taq PCR buffer, $2.5 \mathrm{mM} \mathrm{MgCl} 2,0.4 \mathrm{mM}$ of each dNTP, $1.25 \mathrm{U}$ Maxima Hot Start Taq DNA Polymerase (all from Thermo Scientific ${ }^{\mathrm{TM}}$, Thermo Fisher Scientific, Vilnius, Lithuania), $1 \mu \mathrm{M}$ of each primer, and 10-20 ng of bisulfite-treated DNA. Reaction conditions were optimized before the study and included $35-38$ cycles with primer annealing step $55-62{ }^{\circ} \mathrm{C}$ for $45 \mathrm{~s}$ (Table S2). Methylation-positive (MC), methylation-negative, and non-template controls (NTC) were included in each MSP assay.

\subsection{Quantitative Methylation Analysis}

Target-specific quantitative MSP (QMSP) primers and hydrolysis probes for $P R K C B$, CCDC181, ADAMTS12, and NAALAD2 were designed using MethPrimer software v1.0 (http:/ / www.urogene.org/methprimer; accessed 1 March 2018) [15]. Stably expressed $A C T B$ served as a normalization gene, which was included in each assay to remove the non-biological variation. Primer sequences are provided in Table S3. QMSP was performed with technical triplicates for each set of primers. The reaction mixture of a $20-\mu \mathrm{L}$ final volume contained $1 \times$ TaqMan ${ }^{\circledR}$ Universal Master Mix II, no UNG (Applied Biosystems ${ }^{\mathrm{TM}}$ ), $300 \mathrm{nM}$ of each primer, $50 \mathrm{nM}$ of TaqMan probe, and $10 \mathrm{ng}$ of bisulfite-converted DNA. QMSP was performed using the Mx3005P qPCR System (Agilent Technologies) under the following regime: $95^{\circ} \mathrm{C}$ for $10 \mathrm{~min}$ followed by 50 cycles of $95^{\circ} \mathrm{C}$ for $15 \mathrm{~s}$ and $60{ }^{\circ} \mathrm{C}$ for $1 \mathrm{~min}$. When routinely included MCs gave a positive signal, a run was considered valid, and there was no amplification in NTC wells. The methylation level of an individual gene was estimated based on $\Delta \Delta \mathrm{Cq}$ algorithm and expressed as a percentage of the MC. Samples with a methylation level of $>0.1 \%$ were considered methylated when analyzed qualitatively.

\subsection{RNA Extraction and Gene Expression Analysis by RT-qPCR}

Total RNA samples, prepared during our previous study [10] from the same patient cohort, were used for target gene expression analysis by quantitative PCR (qPCR). Briefly, the mirVana ${ }^{\mathrm{TM}}$ miRNA Isolation Kit (Ambion, Thermo Fisher Scientific, Foster City, CA, USA) was used for total RNA extraction. Only samples having high purity parameters and RNA integrity number (RIN) $\geq 7$, as measured using the 2100 Bioanalyzer (Agilent Technologies), were submitted for further analysis (Table S1). Reverse transcription (RT) was carried out using a High Capacity cDNA Reverse Transcription Kit with RNase Inhibitor (Applied Biosystems ${ }^{\mathrm{TM}}$ ), and $250 \mathrm{ng}$ of RNA as a template.

Expression of genes PRKCB, CCDC181, ADAMTS12, NAALAD2, ZMIZ1, and endogenous control HPRT1 was evaluated using primers and probes from Applied Biosystems (TaqMan ${ }^{\circledR}$ Gene Expression Assays). RT-qPCR was performed with technical triplicates for each set of primers/probes. The reaction mixture of a $20-\mu \mathrm{L}$ final volume contained $1 \times$ TaqMan ${ }^{\circledR}$ Universal Master Mix II, no UNG (Applied Biosystems ${ }^{\mathrm{TM}}$ ), $0.6 \times$ of TaqMan ${ }^{\circledR}$ assay, and $2 \mu \mathrm{L}$ of cDNA, under the following conditions: $95^{\circ} \mathrm{C}$ for $10 \mathrm{~min}$, followed by 
40 cycles of $95^{\circ} \mathrm{C}$ for $15 \mathrm{~s}$ and $60^{\circ} \mathrm{C}$ for $1 \mathrm{~min}$. Real-time amplification was achieved using the Mx3005P qPCR System (Agilent Technologies). Multiple NTCs were included in each RT-qPCR run. Data pre-processing was performed with GenEx v6.0.1 software (MultiD Analyses AB, Göteburg, Sweden): target gene expression levels were normalized with HPRT1. The obtained cycle of quantification differences ( $\triangle \mathrm{Cq}$ values) was converted to an arbitrary linear scale $\left(2^{-\Delta \mathrm{Cq}}\right)$. The obtained relative gene expression values were used for further analysis.

\subsection{Statistical Analysis}

Statistical analyses were performed using STATISTICA ${ }^{\mathrm{TM}}$ v8.0 (StatSoft, Tulsa, OK, USA) and MedCalc ${ }^{\circledR}$ v12.7 (MedCalc Software, Ostend, Belgium). The methylation frequency was calculated as the percentage of samples in which methylation of a particular gene was detected. Student's $t$-test or Mann-Whitney U test was used to compare quantitative variables, while a 2-sided Fisher's exact test was applied to compare categorical variables. Pearson (RP) and/or Spearman's (RS) rank correlation coefficients were calculated to test the associations between two variables. For BCR-free analysis, Kaplan-Meier curves with the log-rank test and Cox proportional hazards models were used. Parametric tests were applied for the analysis of TCGA data. Results were considered statistically significant when the $p$-value was $<0.0500$.

\section{Results}

\subsection{Microarray-Based DNA Methylation Analysis}

The global DNA methylation profile was analyzed in nine cancerous and NPT samples (Figure 1A-D) to evaluate the extent of epigenetic alterations in PCa. Significant methylation differences with FC $\geq 1.2(p<0.0500)$ were detected in 6899 genes, where 4227 $(61.3 \%)$ and $3268(47.4 \%)$ genes were hypermethylated and hypomethylated, respectively, including $596(8.6 \%)$ overlapped genes (Figure 1A). The number of hypermethylated genes in promoter regions was much higher than hypomethylated changes $(72.8 \%$ vs. $29.5 \%$, respectively, with $2.3 \%$ overlap). Meanwhile, both alterations were similarly common in intragenic loci ( $55.8 \%$ vs. $51.0 \%$, respectively, with $6.8 \%$ overlap; Figure $1 C, D)$.

Less numerous DNA methylation differences were observed for patients with and without BCR (Figure 1B). Of 1804 genes with significant methylation differences, 969 $(53.7 \%)$ and $868(48.1 \%)$ genes were hypermethylated and hypomethylated, respectively, including $33(1.8 \%)$ overlapped genes. Increase and decrease of methylation levels were similar in both promoter ( $53.2 \%$ vs. $47.6 \%$, respectively, with $0.9 \%$ overlap) and intragenic regions ( $44.8 \%$ vs. $56.8 \%$, respectively; Figure $1 C, D)$.

Hypermethylation of 411 overlapped genes was detected comparing PCa vs. NPT and BCR positive vs. negative cases, while 291 genes were hypomethylated in both comparisons. Interestingly, some of these genes demonstrated hypermethylation and hypomethylation of different loci (Figure 1C,D). The top 50 genes with the most significant differences are provided in Table S4.

According to GSEA analysis, genes participating in cell cycle regulation, estrogen response, and apical junction were among the most significantly methylated in PCa vs. NPT (Figure 1E). Increased methylation levels were the most significantly different among genes participating in response to ultraviolet exposure and epithelial-mesenchymal transition. Hypomethylation was the most commonly detected in genes responsible for mitotic spindle formation and estrogen response. Similar genes were hypermethylated comparing BCRpositive vs. negative $\mathrm{PCa}$ cases, while genes associated with androgen and estrogen response, and hypoxia, were the most commonly hypomethylated (Figure 1E). 
A

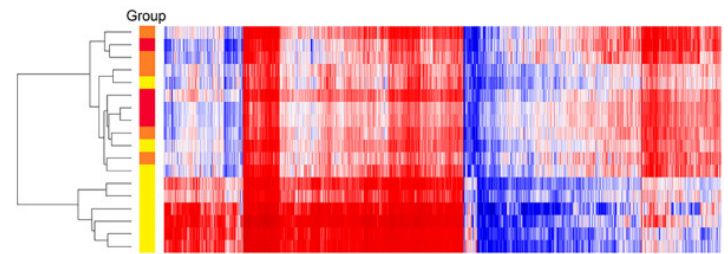

B

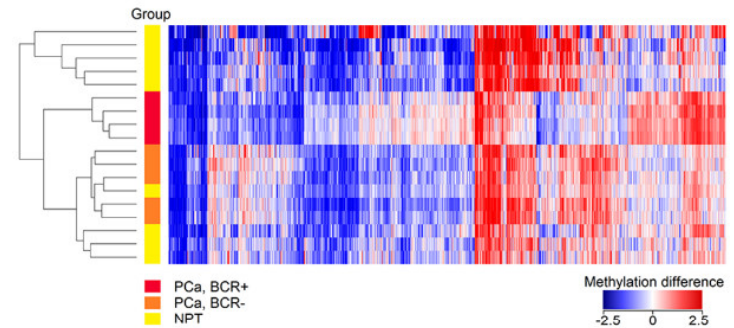

C

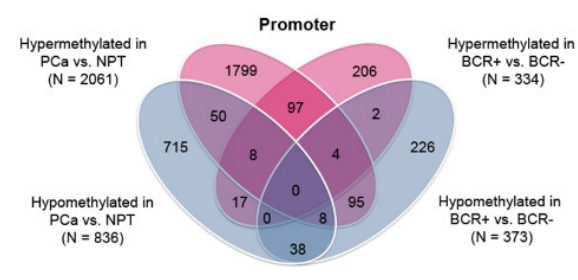

D

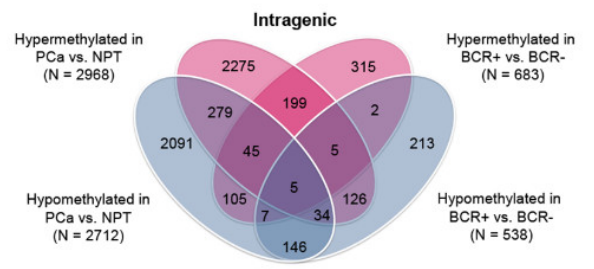

E

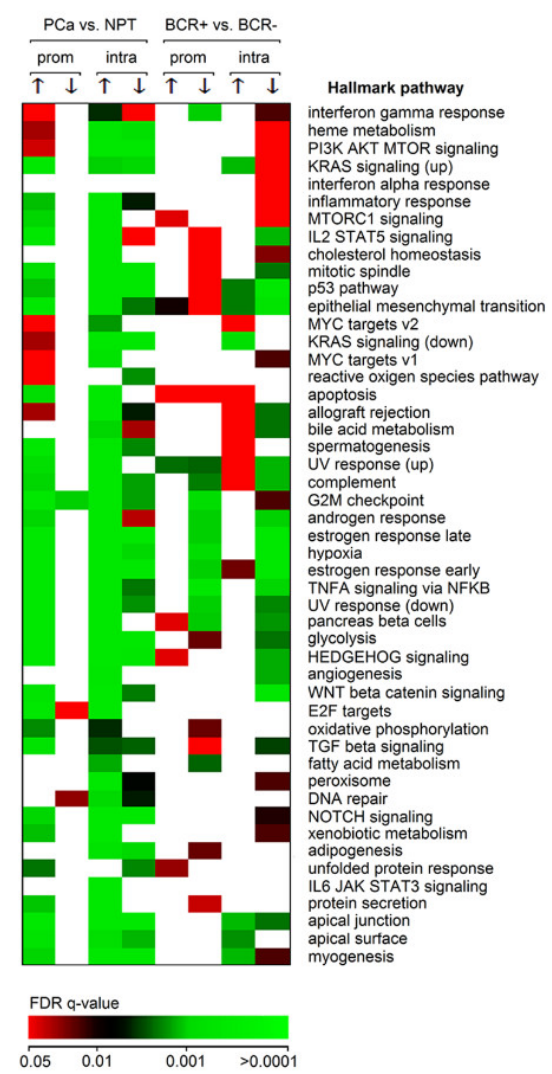

Figure 1. Microarray-based DNA methylation analysis. (A) DNA methylation differences between cancerous and NPT; (B) DNA methylation differences according to biochemical disease recurrence; (C,D) Venn diagrams of methylated genes in promoter and intragenic regions, according to tissue histology and BCR status; (E) Gene set enrichment analysis (GSEA) of differentially methylated genes. The color scale is the same for both heatmaps. The collection of Hallmark gene sets as defined in MSigDB were selected for GSEA. The empty cells in the graph represent insignificant q-values. $\mathrm{PCa}-$ prostate cancer; NPT_-noncancerous prostate tissue; $\mathrm{BCR}+$ / - - biochemical disease recurrence, i.e., yes $(+)$ or no (-); prom-promoter regions, intra—intragenic region; up/down arrows-gain/loss of methylation.

\subsection{Promoter Methylation Analysis}

Ten genes (namely, ADAMTS12, CCDC181, CD44, EPAS1, FILIP1L, KCTD8, NAALAD2, NEK9, PRKCB, and ZMIZ1) were selected for further validation in $151 \mathrm{PCa}, 51 \mathrm{NPT}$, and $17 \mathrm{BPH}$ samples (Table S2), based on the methylation differences in promoter regions according to prostate tissue histology (ADAMTS12, CCDC181, CD44, FILIP1L, KCTD8, NAALAD2, PRKCB, ZMIZ1) or BCR status (EPAS1, FILIP1L, NEK9, PRKCB, ZMIZ1). Frequent methylation of ADAMTS12, CCDC181, FILIP1L, NAALAD2, PRKCB, and ZMIZ1 (up to $90.7 \%$ ), as well as less common of CD44 and KCTD8 (up to $34.4 \%$ ), was identified in PCa, which significantly differed from NPT $(0-35.3 \%)$ and BPH samples $(0 \%)$ (all $p<0.0500$; Figure 2A). Further investigation of EPAS1 and NEK9 was discontinued due to the lack of aberrant methylation events at the promoter regions in a subset of PCa and NPT samples. Quantitative analysis showed that methylation levels of ADAMTS12, CCDC181, NAALAD2, and $P R K C B$ were significantly higher in randomly selected 15 PCa samples than $15 \mathrm{BPH}$ samples (all $p<0.0500$; Figure 2B). 
A

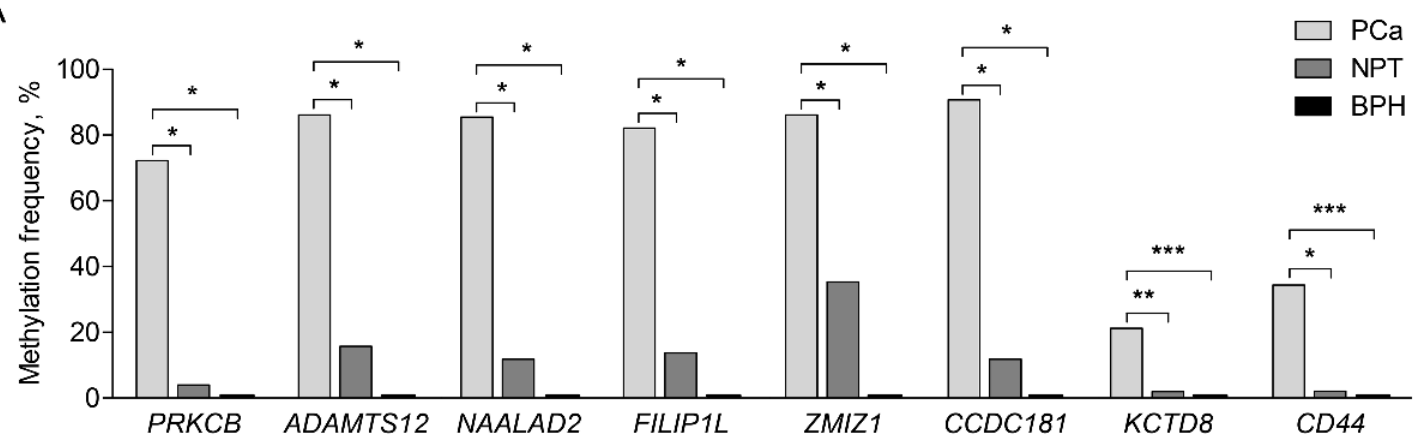

B

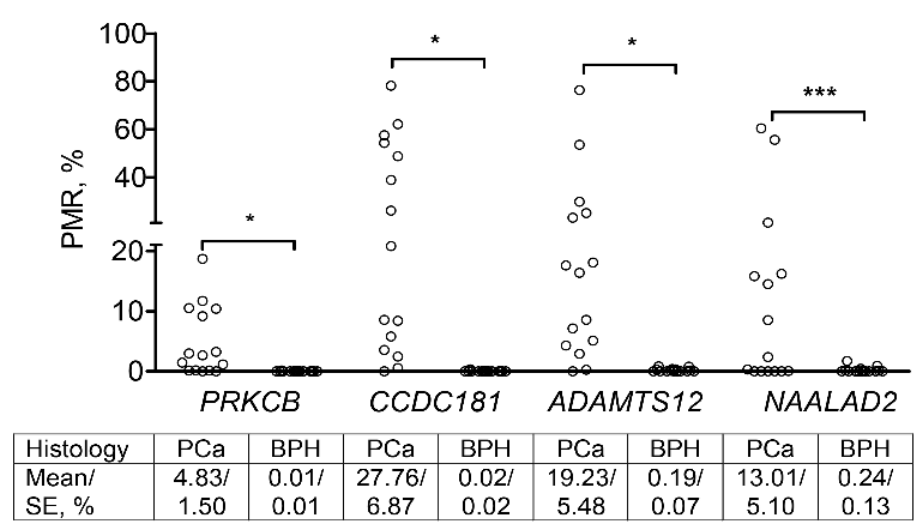

Figure 2. Promoter methylation analysis of the selected target genes in prostate tissue samples. (A) Promoter methylation frequencies of the analyzed genes (MSP data); (B) Methylation intensity values as a percentage of methylated reference (PMR) of genes PRKCB, CCDC181, ADAMTS12, and NAALAD2 (QMSP data). Mean PMR values with standard error of the mean (SE) are given below for each group. PCa—prostate cancer, NPT—noncancerous prostate tissue, BPH—benign prostatic hyperplasia. Statistically significant $p$-values are marked in bold. ${ }^{*}-p \leq 0.0001,{ }^{* *}-p<0.001,{ }^{* * *}-p<0.05$.

To confirm our findings, 333 PCa cases from PRAD data collection of TCGA were used [9]. In line with our findings, all eight genes demonstrated significantly higher methylation levels in PCa than NPT (all $p<0.0001$; Figure S1). Additionally, CD44 (median $\beta$-value 0.26 ) and KCTD8 (median $\beta$-value 0.15 ) were characterized by lower methylation levels in cancerous prostate tissue as compared to other genes (median $\beta$-values $\geq 0.48$ ). In contrast, methylation levels of FILIP1L were relatively high in cancerous and NPT with median $\beta$-values of 0.89 and 0.84 , respectively (Figure S1).

All selected genes were further analyzed according to clinico-pathological characteristics of the disease. Methylation frequency of NAALAD2, PRKCB and ZMIZ1 significantly increased as the ISUP grade group increased, while KCTD 8 and ZMIZ1 were more frequently methylated in locally advanced disease $(\geq \mathrm{pT} 3)$ (all $p<0.0500)$. Besides, ADAMTS12, CD44, $K C T D 8$, and $P R K C B$ were more commonly methylated in tumors harboring gene fusion between TMPRSS2 and ERG (all $p<0.0500$; Table S5). No associations between promoter methylation and PSA level, prostate volume, or the patient's age were detected.

\subsection{Gene Expression Analysis}

Based on promoter methylation frequencies and correlations with clinico-pathological variables, ADAMTS12, CCDC181, NAALAD2, PRKCB, and ZMIZ1 were selected for further expression analysis. RNA with suitable quality for molecular analysis was available from $81 \mathrm{PCa}, 25 \mathrm{NPT}$, and $17 \mathrm{BPH}$ samples from the same patient cohort (Table S1). ADAMTS12, CCDC181, NAALAD2, and PRKCB demonstrated significantly lower expression levels in cancerous prostate tissue than NPT and BPH samples (all $p<0.0500$; Figure 3A-D). The expression level of ZMIZ1 was also lower in cancerous than in NPT, but higher than in BPH (all $p<0.0500$; Figure 3E). Furthermore, lower expression levels of ADAMTS12, CCDC181, 
NAALAD2, and $P R K C B$ in cancerous tissue correlated with promoter methylation status (all $p \leq 0.0001$; Figure 3F-I), while no such association was demonstrated by ZMIZ1 (Figure 3J).

A

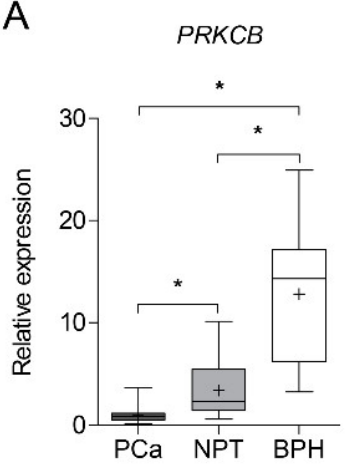

D

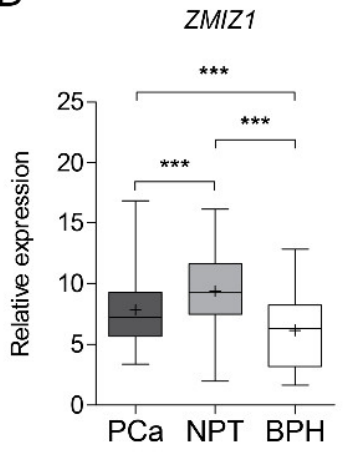

B

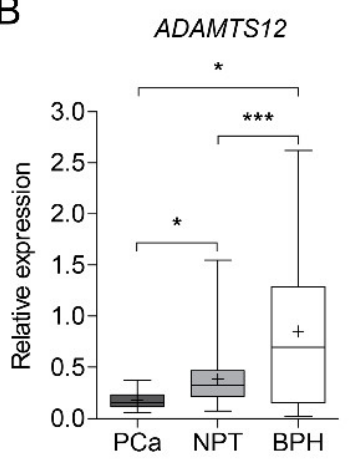

$\mathrm{E}$

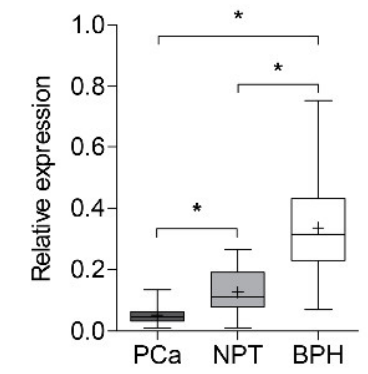

C

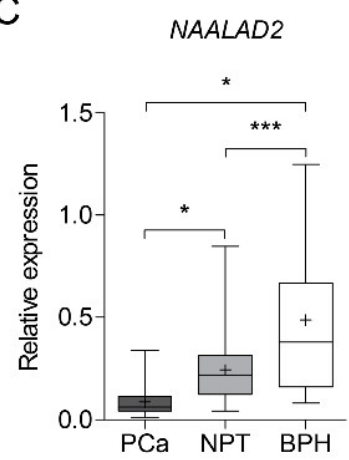

$\mathrm{F}$

$P R K C B$

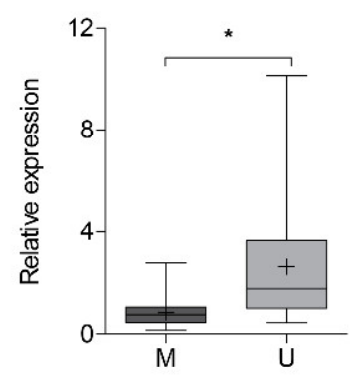

$\mathrm{J}$
G

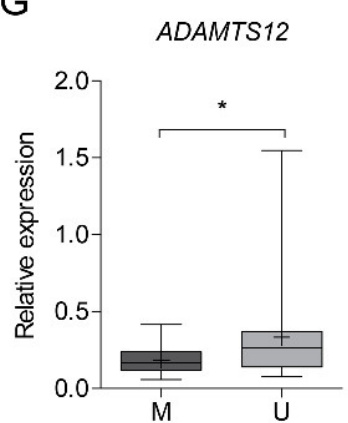

$\mathrm{H}$

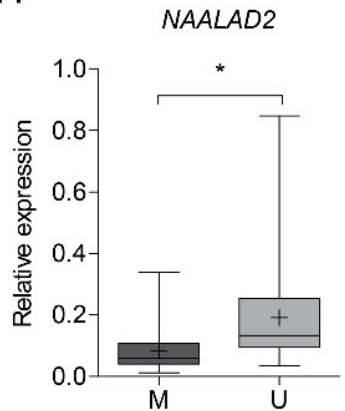

I

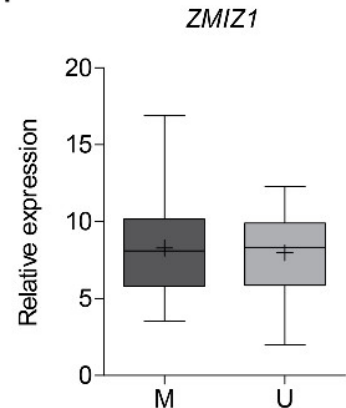

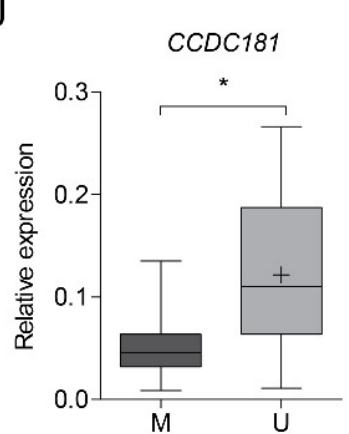

Figure 3. Expression of ADAMTS12, CCDC181, NAALAD2, PRKCB, and ZMIZ1 in cancerous and noncancerous prostate tissue. (A-E) Relative expression of selected genes in prostate cancer (PCa), noncancerous prostate tissue (NPT), and benign prostatic hyperplasia (BPH) samples; (F-J) Relative expression of selected genes in PCa according to methylation status, i.e., methylated (M) or unmethylated (U; MSP data). The boxplot depicts Q1-Q3 quartile where the line in the box indicates the median and the plus sign indicates the mean. Significant $p$-values are marked in bold. ${ }^{*}-p \leq 0.0001,{ }^{* *}-p<0.05$.

Consistent with our findings, significantly lower expression levels of ADAMTS12, CCDC181, NAALAD2, as well as PRKCB were identified in PCa as compared to NPT in the PRAD cohort (all $p<0.0500)$, except for ZMIZ1 ( $p>0.0500$; Figure S2). Moreover, $C C D C 181, P R K C B$, and ZMIZ1 demonstrated significantly lower expression levels in cancerous prostate tissue with higher methylation intensity (all $p<0.0500$ ), while no statistically significant results were observed for ADAMTS12 and NAALAD2 (all $p>0.0500$; Figure S3). 
In our cohort, lower expression levels of CCDC181 and NAALAD2 significantly correlated with higher postoperative ISUP grade group ( $p=0.0016$ and $p=0.0015$; Table S5), while the higher expression level of CCDC181 was specific for tumors harboring TMPRSS2$E R G$ gene fusion $(p=0.0136)$. No associations between the expression level of any gene and clinico-pathological characteristics, such as pT stage, PSA level, and prostate volume, were observed. However, the expression level of NAALAD2 revealed a positive correlation with patients' age (RS $=0.27, p=0.0153$; Table S5).

\subsection{Survival Analysis}

BCR-free survival analysis was performed to investigate the prognostic potential of PCa-specific genes. Aberrant promoter methylation of ADAMTS12, NAALAD2 and PRKCB was more frequent in patients undergoing $B C R$ than in patients with no biochemical relapse $(p=0.0036, p=0.0019$ and $p=0.0039$, respectively; Figure 4A). Comparison of Kaplan-Meier curves revealed significantly lower BCR-free survival rates in PCa patients with aberrant promoter methylation of ADAMTS12, NAALAD2, and PRKCB, as well as ZMIZ1 ( $p=0.0023$, $p=0.0025, p=0.0051$ and $p=0.0370$, respectively; Figure 4B-E). Meanwhile, no statistically significant differences were observed for other investigated biomarkers (not shown).

The prognostic value of ADAMTS12, NAALAD2, and PRKCB was also supported by univariate and multivariate Cox proportional hazard analyses. However, only NAALAD2 showed significant prognostic value at the gene expression level (all models $p<0.0500$; Table S6). Forward stepwise variable selection revealed that methylated NAALAD2 or $P R K C B$ together with $\mathrm{pT}$ stage or ISUP grade group outperformed the prognostic power of clinico-pathological characteristics alone (all models $p<0.0001$ ). Besides, the combination of $P R K C B$ promoter methylation status with PSA significantly predicted BCR-free survival, although PSA lacked significance as an independent factor (models $p=0.0008$ and $p>0.0500)$. Methylation of ADAMTS12, NAALAD2, and PRKCB combined with TMPRSS2$E R G$ fusion also demonstrated a significant prognostic potential for BCR-free survival (all $p<0.0500$ ). Selected multivariate Cox models are provided in Table S7.

In TCGA data analysis, PCa cases with ISUP grade group 5, metastatic disease, previous active surveillance, or neoadjuvant therapy were excluded from further analysis to better match our cohort. Similar to our findings, methylation levels of NAALAD2 and $P R K C B$ combined with the $\mathrm{pT}$ stage or ISUP grade group revealed significant prognostic value (all models $p<0.0500$; Table S7). However, in univariate analysis, methylation levels of the selected genes were not associated with BCR status, while decreased expression of ADAMTS12 and PRKCB demonstrated prognostic potential for BCR-free survival (all $p<0.0500$; Table S6). 


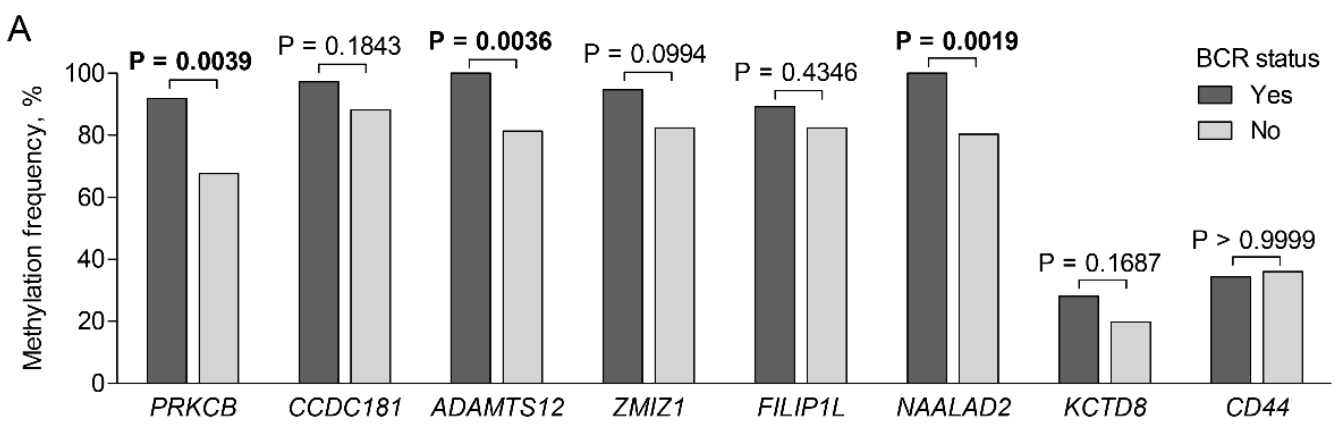

B

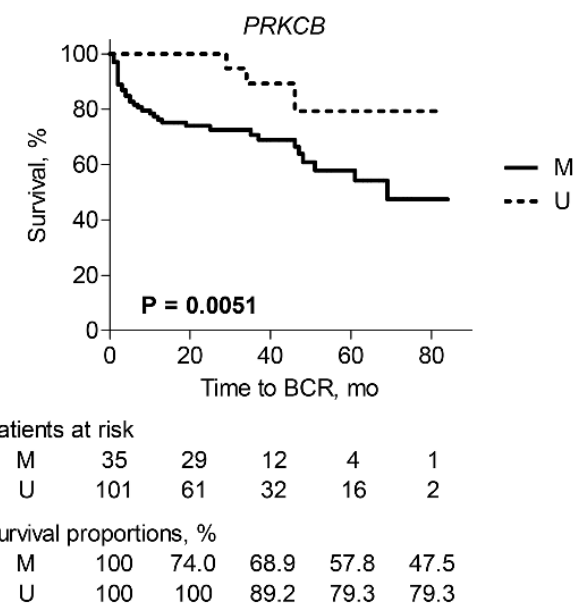

D

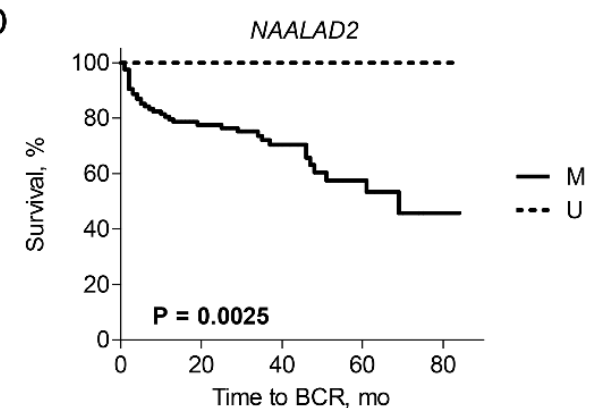

C

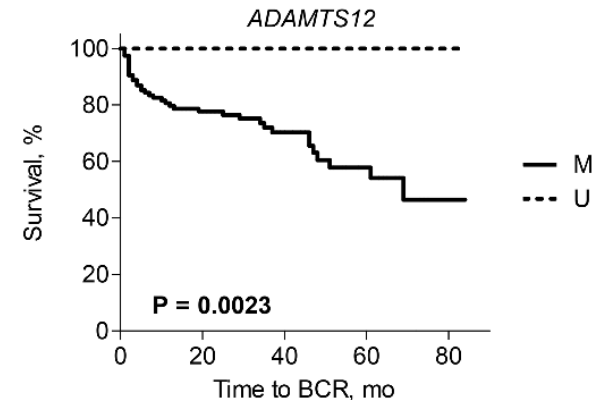

$\mathrm{E}$

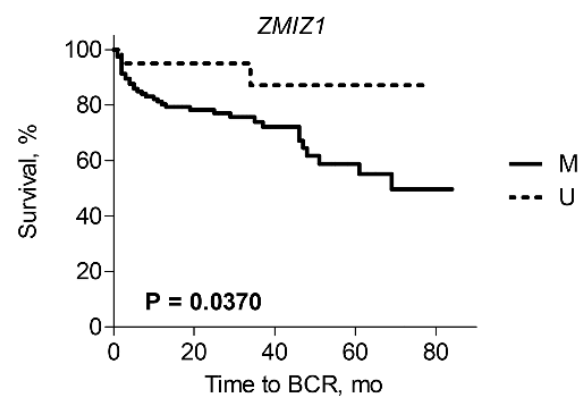

\begin{tabular}{cccccc}
\multicolumn{5}{c}{ Patients at risk } \\
M & 116 & 72 & 34 & 16 & 3 \\
U & 20 & 18 & 10 & 4 & 0 \\
\multicolumn{5}{l}{ Survival proportions, \% } \\
M & 100 & 78.3 & 72.1 & 58.8 & 49.6 \\
U & 100 & 100 & 84.5 & 87.1 & 87.1
\end{tabular}

Figure 4. Promoter methylation status of selected genes in prostate cancer (PCa) according to biochemical recurrence (BCR) status. (A) Promoter methylation frequencies of the analyzed genes; (B-E) Kaplan-Meier curves according to methylation of ADAMTS12, NAALAD2, PRKCB, and ZMIZ1. $\mathrm{M}-$ methylated, $\mathrm{U}$ - unmethylated. Significant $p$-values are marked in bold.

\section{Discussion}

Recent progress in genomic technologies, such as microarrays and next-generation sequencing, have opened new possibilities for researchers to conduct genome-wide methylation analysis in various malignancies, primarily focusing on novel biomarkers that could eliminate the limitations of currently used ones. During recent years various studies have made important insight into DNA methylation profile of PCa and identified a large number of novel molecular biomarkers. However, only a few of them have been transferred into clinical practice so far $[5,16]$. 
In the current study, PCa methylome data, obtained from well-characterized PCa cohort and matched NPT samples using microarray [10], was applied to screen novel diagnostic and prognostic PCa biomarkers. In agreement with other authors $[5,6,16,17]$, comparing cancerous tissues with NPT and cases with and without BCR, different methylation of various promoters was identified, and the changes affected both intra- and intergenic loci [5,7]. Although microarray design mainly covered various promoter CpG islands regardless of their co-localization with annotated regulatory regions, further validation of selected genes was based on promoter-associated methylation differences.

The majority of the genes reported in our study have been poorly investigated in PCa or other types of malignancies. Some of the most established PCa genes, such as $R A R B$ and RASSF1, were differently methylated in our cohort. However, like APC and GSTP1, others did not show any significant differences in methylation, a phenomenon also reported in other studies $[7,16]$. Both biological and technical perspectives could at least partially explain all these contradictory results. PCa is a particularly heterogeneous and multifocal disease, leading to differences in biological samples with various cancerous cellularity and aggressiveness. Technical aspects of the procedure, such as different material collection and different processing, storage, research methods, and cut-off interpretation, are also of utmost importance and may have an immense impact on DNA methylation differences between various studies.

Nonetheless, several previously reported genes, such as CCDC181 [18] and SEPT9 [19], demonstrated one of the most significant methylation differences in PCa. A 4-gene test, including CCDC181, has been proposed to improve PCa detection through epigenetic field effect, where histologically normal prostate tissues in the vicinity of a PCa harbors distinct epigenetic alterations [20]. In agreement, CCDC181 was the most frequently methylated gene $(90.7 \%)$ of the 10 -gene set in our study, with up to $100 \%$ specificity for PCa. In the study by Haldrup et al., methylation of CCDC181 demonstrated independent value to predict BCR-free survival [18]. However, according to our analysis, the prognostic potential of this gene was not confirmed in our and TCGA cohorts.

The significant role of ZMIZ1 has been described in PCa progression to a castrationresistant form of the disease through recruitment on the promoter of KLK3 gene [21]. Similarly, promoter methylation and/or downregulated expression of FILIP1L have been associated with an aggressive form of the disease [22,23]. According to our data, no significant differences between FILIP1L and ZMIZ1 methylation frequencies and BCR were observed, which could be explained by the stringent inclusion of low-intermediate risk localized PCa cases into our cohort. However, ZMIZ1 was more commonly methylated in tumors with advanced pT stage and higher ISUP grade group.

Among the large number of differentially methylated genes identified in the Lithuanian cohort, promoter methylation of ADAMTS12, NAALAD2, and PRKCB had not only high sensitivity $(86.1 \%)$ and specificity for $\mathrm{PCa}(\geq 84.3 \%)$ but also revealed potential prognostic value. According to our knowledge, these three genes have never been investigated as DNA methylation biomarkers for PCa.

Methylation of $P R K C B$ has been investigated in few types of malignancies, where a positive correlation was identified between methylation of two $\mathrm{CpGs}$ and gene expression by analyzing the TCGA dataset of lung adenocarcinoma [24]. In our analysis, PRKCB methylation status was strongly associated with decreased gene expression in Lithuanian and TCGA PCa cohorts. Supporting this idea, treatment with demethylating agents has been previously reported to restore $P R K C B$ expression [25]. However, this is contradictory data to other studies, reporting up-regulation of $P R K C B$ in $\mathrm{PCa}$ [26] and its oncogenic role through activated angiogenesis and increased cell proliferation [27]. Furthermore, Metzger et al. have demonstrated that $P R K C B$ controls androgen receptor (AR)-dependent gene expression during PCa progression through interaction with KDM1A and AR [26]. In our study, promoter methylation of $P R K C B$ was predictive of BCR-free survival as an independent factor and in combinations with clinico-pathological characteristics. Because 
$P R K C B$ encodes several distinct isoforms, the role of promoter methylation of $P R K C B$ in $P C a$ requires further investigations.

Despite belonging to the same NAALADase gene family as the prostate-specific membrane antigen gene (PSMA), little is known about NAALAD2. There is a lack of data regarding promoter methylation of the gene. The aberrant methylation of ADAMTS12, as a novel tumor suppressor gene, has been previously investigated only in colon cancer, which was associated with disease progression [28]. In our study, promoter methylation of ADAMTS12 and NAALAD2 was predictive of BCR-free survival independently or in combination with clinico-pathological characteristics. Besides, together with $P R K C B$, these potential epigenetic biomarkers composed a multivariate model of molecular-only covariates for the prognosis of BCR-free survival. Therefore, the 3-gene methylation assay could provide valuable diagnostic information about the aggressive form of the disease and guide to radical treatment options to obtain the best possible oncological outcomes and assist in BCR prognosis making that could lead to early adjuvant or salvage treatment options. However, further investigations in independent cohorts are needed to confirm our findings before implementing these specific biomolecular markers into clinics.

\section{Conclusions}

Three novel PCa-specific biomarkers were identified through genome-wide screening of DNA methylation changes in PCa. Promoter methylation of ADAMTS12, NAALAD2, and $P R K C B$ showed high sensitivity and specificity for PCa. The methylation status of investigated genes was also independently predictive for poor BCR-free survival and had even higher prognostic power in models with the established clinico-pathological characteristics. Future studies in larger cohorts are needed to further assess the proposed biomarker assay's clinical value and evaluate its potential for noninvasive testing in body fluids, like urine or blood.

\section{Patents}

Resulting to this work, a patent application "Characterization of Prostate Cancer Using DNA Methylation Assay System" No. PCT/IB2019/056204 was submitted on 18 September 2019, which latter was licensed to "ThermoPharma Baltic".

Supplementary Materials: The following are available online at https:/ /www.mdpi.com/article/10 $.3390 /$ ijms22116091/s1.

Author Contributions: K.D. performed methylome profiling, gene expression experiments, statistical analysis, supervised DNA methylation experiments and participated in the original draft preparation; A.B. collected and analyzed clinical data, participated in the original draft preparation and final corrections; K.Z. Participated in manuscript corrections; I.R. performed DNA methylation analysis and participated in gene expression experiments; J.R.L. coordinated sample and data collection; A.U. implemented patient selection and follow-up; F.J. supplied the samples and clinical data, reviewed and edited the manuscript, was responsible for the granting; S.J. conceived and designed the study, coordinated research activities, as well as reviewed and edited the manuscript. All authors have read and agreed to the published version of the manuscript.

Funding: The present study was funded by grant No. SEN-9/2016 from the Research Council of Lithuania (RCL). Sample collection was also partially supported by grant No. LIG-14/2012 from RCL.

Institutional Review Board Statement: The study was conducted according to the guidelines of the Declaration of Helsinki and approved by the Lithuanian Bioethics Committee (2007-11-23 Nr.:50 and 2011-09-07 Nr.:6B-11-275).

Informed Consent Statement: Written informed consent was obtained from all subjects involved in the study.

Data Availability Statement: All data supporting the results reported in the article is available from the corresponding author upon a reasonable request. 
Conflicts of Interest: K.D. and S.J. are inventors of the patent application for DNA methylation biomarkers (application No. PCT/IB2019/056204; filed by Vilnius University). JSC "ThermoPharma Baltic" has licensed the patent. K.D. is employed at JSC "ThermoPharma Baltic". The remaining authors declare that they have no conflict of interest. The funders had no role in the design of the study; in the collection, analyses, or interpretation of data; in the writing of the manuscript, or in the decision to publish the results.

\section{References}

1. Ferlay, J.; Colombet, M.; Soerjomataram, I.; Mathers, C.; Parkin, D.M.; Piñeros, M.; Znaor, A.; Bray, F. Estimating the global cancer incidence and mortality in 2018: GLOBOCAN sources and methods. Int. J. Cancer 2019, 144, 1941-1953. [CrossRef] [PubMed]

2. Cooperberg, M.R.; Broering, J.M.; Kantoff, P.W.; Carroll, P.R. Contemporary Trends in Low Risk Prostate Cancer: Risk Assessment and Treatment. J. Urol. 2007, 178, S14-S19. [CrossRef] [PubMed]

3. Sanda, M.G.; Dunn, R.L.; Michalski, J.; Sandler, H.M.; Northouse, L.; Hembroff, L.; Lin, X.; Greenfield, T.K.; Litwin, M.S.; Saigal, C.S.; et al. Quality of Life and Satisfaction with Outcome among Prostate-Cancer Survivors. N. Engl. J. Med. 2008, 358, $1250-1261$. [CrossRef]

4. Park, J.Y. Promoter Hypermethylation as a Biomarker in Prostate Adenocarcinoma. Cancer Epigenetics 2014, 1238, 607-625. [CrossRef]

5. Kim, J.H.; Dhanasekaran, S.M.; Prensner, J.; Cao, X.; Robinson, D.; Kalyana-Sundaram, S.; Huang, C.; Shankar, S.; Jing, X.; Iyer, M.; et al. Deep sequencing reveals distinct patterns of DNA methylation in prostate cancer. Genome Res. 2011, 21, 1028-1041. [CrossRef]

6. Kobayashi, Y.; Absher, D.M.; Gulzar, Z.G.; Young, S.R.; McKenney, J.K.; Peehl, D.M.; Brooks, J.D.; Myers, R.M.; Sherlock, G. DNA methylation profiling reveals novel biomarkers and important roles for DNA methyltransferases in prostate cancer. Genome Res. 2011, 21, 1017-1027. [CrossRef] [PubMed]

7. Kron, K.; Pethe, V.; Briollais, L.; Sadikovic, B.; Ozcelik, H.; Sunderji, A.; Venkateswaran, V.; Pinthus, J.; Fleshner, N.; Van Der Kwast, T.; et al. Discovery of Novel Hypermethylated Genes in Prostate Cancer Using Genomic CpG Island Microarrays. PLoS ONE 2009, 4, e4830. [CrossRef] [PubMed]

8. Mahapatra, S.; Klee, E.W.; Young, C.Y.; Sun, Z.; Jimenez, R.E.; Klee, G.G.; Tindall, D.J.; Donkena, K.V. Global Methylation Profiling for Risk Prediction of Prostate Cancer. Clin. Cancer Res. 2012, 18, 2882-2895. [CrossRef]

9. The Cancer Genome Atlas Research Network. The Molecular Taxonomy of Primary Prostate Cancer. Cell 2015, 163, 1011-1025. [CrossRef]

10. Daniunaite, K.; Dubikaityte, M.; Gibas, P.; Bakavicius, A.; Lazutka, J.R.; Ulys, A.; Jankevicius, F.; Jarmalaite, S. Clinical significance of miRNA host gene promoter methylation in prostate cancer. Hum. Mol. Genet. 2017, 26, 2451-2461. [CrossRef]

11. Sabaliauskaite, R.; Jarmalaite, S.; Petroska, D.; Dasevicius, D.; Laurinavicius, A.; Jankevicius, F.; Lazutka, J.R. Combined analysis of TMPRSS2-ERG and TERT for improved prognosis of biochemical recurrence in prostate cancer. Genes Chromosomes Cancer 2012, 51, 781-791. [CrossRef] [PubMed]

12. Moul, J.W. Prostate specific antigen only progression of prostate cancer. J. Urol. 2000, 163, 1632-1642. [CrossRef]

13. Subramanian, A.; Tamayo, P.; Mootha, V.K.; Mukherjee, S.; Ebert, B.L.; Gillette, M.A.; Paulovich, A.; Pomeroy, S.L.; Golub, T.R.; Lander, E.S.; et al. Gene set enrichment analysis: A knowledge-based approach for interpreting genome-wide expression profiles. Proc. Natl. Acad. Sci. USA 2005, 102, 15545-15550. [CrossRef]

14. Liberzon, A.; Birger, C.; Thorvaldsdottir, H.; Ghandi, M.; Mesirov, J.P.; Tamayo, P. The Molecular Signatures Database (MSigDB) hallmark gene set collection. Cell Syst. 2015, 1, 417-425. [CrossRef]

15. Li, L.-C.; Dahiya, R. MethPrimer: Designing primers for methylation PCRs. Bioinformatics 2002, 18, 1427-1431. [CrossRef] [PubMed]

16. Devaney, J.M.; Wang, S.; Funda, S.; Long, J.; Taghipour, D.J.; Tbaishat, R.; Furbert-Harris, P.; Ittmann, M.; Kwabi-Addo, B. Identification of novel DNA-methylated genes that correlate with human prostate cancer and high-grade prostatic intraepithelial neoplasia. Prostate Cancer Prostatic Dis. 2013, 16, 292-300. [CrossRef]

17. Kirby, M.K.; Ramaker, R.C.; Roberts, B.S.; Lasseigne, B.N.; Gunther, D.S.; Burwell, T.C.; Davis, N.S.; Gulzar, Z.G.; Absher, D.M.; Cooper, S.J.; et al. Genome-wide DNA methylation measurements in prostate tissues uncovers novel prostate cancer diagnostic biomarkers and transcription factor binding patterns. BMC Cancer 2017, 17, 273. [CrossRef]

18. Haldrup, C.; Mundbjerg, K.; Vestergaard, E.M.; Lamy, P.; Wild, P.; Schulz, W.A.; Arsov, C.; Visakorpi, T.; Borre, M.; Høyer, S.; et al. DNA Methylation Signatures for Prediction of Biochemical Recurrence After Radical Prostatectomy of Clinically Localized Prostate Cancer. J. Clin. Oncol. 2013, 31, 3250-3258. [CrossRef]

19. Angulo, J.C.; Andrés, G.; Ashour, N.; Sánchez-Chapado, M.; Lopez, J.I.; Ropero, S. Development of Castration Resistant Prostate Cancer can be Predicted by a DNA Hypermethylation Profile. J. Urol. 2016, 195, 619-626. [CrossRef]

20. Møller, M.; Strand, S.H.; Mundbjerg, K.; Liang, G.; Gill, I.; Haldrup, C.; Borre, M.; Høyer, S.; Ørntoft, T.F.; Sørensen, K.D. Heterogeneous patterns of DNA methylation-based field effects in histologically normal prostate tissue from cancer patients. Sci. Rep. 2017, 7, 40636. [CrossRef]

21. Li, X.; Zhu, C.; Yang, N.; Qin, H.; Sun, Z. ZMIZ1 preferably enhances the transcriptional activity of androgen receptor with short poly-glutamine tract. PLOS ONE 2011, 6, e25040. 
22. Desotelle, J.; Truong, M.; Ewald, J.; Weeratunga, P.; Yang, B.; Huang, W.; Jarrard, D. CpG Island Hypermethylation Frequently Silences FILIP1L Isoform 2 Expression in Prostate Cancer. J. Urol. 2013, 189, 329-335. [CrossRef] [PubMed]

23. Kwon, M.; Lee, S.J.; Reddy, S.; Rybak, Y.; Adem, A.; Libutti, S.K. Down-regulation of Filamin A interacting protein 1-like Is associated with promoter methylation and an invasive phenotype in breast, colon, lung and pancreatic cancers. PLoS ONE 2013, 8, e82620. [CrossRef]

24. Liu, S.; Chen, X.; Chen, R.; Wang, J.; Zhu, G.; Jiang, J.; Wang, H.; Duan, S.; Huang, J. Diagnostic role of Wnt pathway gene promoter methylation in non small cell lung cancer. Oncotarget 2017, 8, 36354-36367. [CrossRef]

25. Hagiwara, K.; Ito, H.; Miyata, Y.; Murate, T.; Ohashi, H.; Nagai, H. PROX1 overexpression inhibits protein kinase C beta II transcription through promoter DNA methylation. Genes Chromosome Cancer 2012, 51, 1024-1036. [CrossRef]

26. Metzger, E.; Imhof, A.; Patel, D.; Kahl, P.; Hoffmeyer, K.; Friedrichs, N.; Müller, J.M.; Greschik, H.; Kirfel, J.; Ji, S.; et al. Phosphorylation of histone H3T6 by PKCbeta(I) controls demethylation at histone H3K4. Nature 2010, 464, 792-796. [CrossRef] [PubMed]

27. Kim, J.; Choi, Y.-L.; Vallentin, A.; Hunrichs, B.S.; Hellerstein, M.K.; Peehl, D.M.; Mochly-Rosen, D. Centrosomal PKC $\beta I I$ and Pericentrin Are Critical for Human Prostate Cancer Growth and Angiogenesis. Cancer Res. 2008, 68, 6831-6839. [CrossRef]

28. Moncada-Pazos, A.; Obaya, A.J.; Fraga, M.F.; Viloria, C.G.; Capellá, G.; Gausachs, M.; Esteller, M.; López-Otín, C.; Cal, S. The ADAMTS12 metalloprotease gene is epigenetically silenced in tumor cells and transcriptionally activated in the stroma during progression of colon cancer. J. Cell Sci. 2009, 122, 2906-2913. [CrossRef] [PubMed] 